\title{
Reply to Ropovik and IJzerman's blog post (March 17, 2021)
}

\author{
Spike W. S. Lee, Norbert Schwarz \\ March 17, 2021
}

We thank Ropovik and IJzerman for taking the time to write their blog post that follows up on prior correspondence in BBS (Behavioral and Brain Sciences). Our reading of their blog post is that there are two key aspects of disagreement. First, when implementing a $p$-curve analysis, we consider it crucial to follow all best practices, not just some of them. Second, it is our stance that illustrative evidence in a theory paper should not be treated as if it were comprehensive coverage of all available evidence. We elaborate on both aspects below, the first of which will be quite technical and the second more fundamental.

For clarity of reference, here are the relevant pieces, listed in chronological order:

- Our target article (Lee \& Schwarz, 2021a)

- Ropovik, Sparacio, and IJzerman's commentary (Ropovik et al., 2021)

- Our "authors' response" to all 27 commentaries (Lee \& Schwarz, 2021b) and our response appendix that presents technical details regarding Ropovik et al.

- Ropovik and IJzerman's blog post

- Our present reply to Ropovik and IJzerman's blog post

\section{Follow all best practices in $p$-curve analysis}

In our authors' response, we pointed out that Ropovik et al. drew their conclusion on the basis of a $p$-curve analysis of (i) a small subset of the entire body of experimental research on the psychological consequences and antecedents of physical cleansing (namely, seven out of several hundred effects), which (ii) included only some of the replication studies and (iii) excluded all of the original studies. The procedures they applied to the selected studies (iv) did not follow core steps of best practice recommendations (Simonsohn, Nelson, \& Simmons, 2014b; Simonsohn, Simmons, \& Nelson, 2015), (v) included $p$-values that should be excluded, and (vi) excluded $p$-values that should be included. A detailed discussion of the assorted errors exceeds the space limit of this Response; we provide it in an Appendix available at https://osf.io/sxz97/?view only=630af79ee6c149a5833a4d2fbb4cd560. When best practice recommendations are followed, the effects addressed by Ropovik et al. show evidential value. Such evidential value is found regardless of whether the analysis includes only the replications Ropovik et al. themselves selected or includes the original experiments along with the replications. Ropovik et al.'s errors concerning the identification and specification of the $p$-values also undermine the conclusions from their Monte Carlo simulations.

In their blog post, Ropovik and IJzerman disagree with our characterization. They also suggest that our $p$-curve analysis contains inappropriate changes to theirs. These disagreements boil down to whether one considers it crucial to follow all of the best 
practices in $p$-curve analysis (Simonsohn, Nelson, \& Simmons, 2014; Simonsohn, Simmons, \& Nelson, 2015). We consider it crucial to follow all of them, not just some of them. In so doing, we are perceived by Ropovik and IJzerman as having made inappropriate changes.

At the broadest level, we consider it crucial to have a proper $p$-curve disclosure table. "Editors and reviewers evaluating $p$-curve articles should begin by evaluating the $P$-Curve Disclosure Table... and uncompromisingly demanding that authors follow the guidelines that lead to valid $p$-curves" (Simonsohn et al., 2015, p. 1148). Ropovik et al. provided a table, but it was not a $p$-curve disclosure table, because it missed essential information. As noted in our authors' response, their table

did not "identify the researchers' stated hypothesis and study design" (Step 1 in Simonsohn et al., 2014, p. 540), nor did it "recompute the precise p value(s) based on reported test statistics" (Step 4). "It is essential for $p$-curvers to report how they implemented these steps."

Missing these steps turned out to be consequential.

By failing to identify the researchers' stated hypothesis and study design, Ropovik and IJzerman make erroneous claims about how the results of De Los Reyes et al. (2012) should be handled in a $p$-curve analysis. De Los Reyes et al. hypothesized a three-way interaction in the form of an "attenuation of attenuated interaction" (details available in footnote ${ }^{1}$ ). When this form of three-way interaction is hypothesized by the original researchers, the $p$ curve has to include the three-way interaction effect, as explicitly pointed out by the originators of $p$-curve: "if a $2 \times 2 \times 2$ design tests the prediction that a two-way interaction is itself attenuated, then the $p$ value for the three-way interaction ought to be selected" (Simonsohn et al., 2014, p. 544; also Figure 5, p. 542). The $p$-curver does not get to choose here (details available in footnote ${ }^{2}$ ). We followed the guidelines and appropriately selected the three-way interaction. Ropovik and IJzerman think we made a mistake and should have selected the two-way interaction instead. This is erroneous, because it would have violated

${ }^{1}$ De Los Reyes et al. (2012) hypothesized that the phenomenon of washing away postdecisional dissonance (a two-way interaction between the cleansing vs. no-cleansing manipulation and the evaluation of chosen vs. unchosen options) would be moderated by a construct called compromised decision making or CDM (hence a three-way interaction). Specifically, the phenomenon of washing away postdecisional dissonance was demonstrated by the fact that the classic spreading-of-alternatives effect-the difference in evaluation of chosen vs. unchosen options-was larger in the no-cleansing condition but smaller in the cleansing condition. In technical terms, it was an attenuated interaction. De Los Reyes et al.'s hypothesis was that this attenuated interaction would be larger among participants low in CDM but smaller among participants high in CDM (and that's why they wrote, "To test our main hypotheses, we grouped participants into high-CDM ... and low-CDM... groups"; p. 4). In technical terms, it was an attenuation of attenuated interaction.

2 Simonsohn et al. (2014, p. 543) explained this idea first using the simpler case of a two-way interaction in the form an attenuated interaction: "When the researcher's stated hypothesis is that the interaction attenuates the impact of X on Y (e.g., people always sweat more in summer, but less so indoors), the relevant test is whether the interaction is significant (Gelman \& Stern, 2006), and hence $p$-curve must include only the interaction's $p$ value. Even if the $p$-curver is only interested in the simple effect (e.g., because she is conducting a meta-analysis of this effect), that $p$ value may not be included in $p$-curve if it was reported as part of an attenuated interaction design." Then Simonsohn et al. explained how the same logic extends to more complex cases, as quoted in our main text: "if a $2 \times 2 \times 2$ design tests the prediction that a two-way interaction is itself attenuated, then the $p$ value for the three-way interaction ought to be selected" (p. 544). 
the $p$-curve guidelines and the statistical reasons behind. On the same findings, Ropovik and IJzerman also erroneously claim that "what [Lee and Schwarz] cite in their target article as a replication" was a two-way interaction. We described it as a three-way interaction in our target article (quote available in footnote ${ }^{3}$ ).

Ropovik et al. did not recompute $p$-values based on reported test statistics. Ropovik and IJzerman's defense is that it was a conscious choice, with the goal "to appraise the merits of a finite set of empirical evidence" (italics original). Our disagreement here is that if the goal is to assess the evidence using $p$-curve as a tool, then the tool has to be used properly, following all the guidelines. "Recomputation is necessary" (Simonsohn et al., 2014, p. 542). We diligently implemented this necessary step by recomputing $p$-values based on reported test statistics. Ropovik et al. chose not to. Yet we are being charged for following the guidelines. As Ropovik and IJzerman critique our recomputation, along the way they also argue that we should not have included findings "from unpublished studies with no fullfledged empirical paper available." We disagree. From a meta-analytic perspective, findings from unpublished studies ought to be included: "Inclusive reviews that capture the gray literature and reports in all languages are routinely expected" (Albarracín et al., 2018, Editorial of Psychological Bulletin). Ropovik and IJzerman's critique of using unpublished work to inform one's view is all the more puzzling because Ropovik et al. used unpublished work to inform their views (p. 44): "The specific implementation of bias-correction depends on the analytical context, but for an example of such a workflow, see IJzerman et al. (2020) and Sparacio et al. (2021)," both of which were cited as unpublished.

Ropovik and IJzerman defend the absence of the above essential information (original researchers' stated hypothesis, study design, and recomputed p-values) from Ropovik et al.'s table by arguing that " $[\mathrm{t}]$ he point of a disclosure table is to identify the target effect in a study to ensure that the synthesized effect was the focal effect of the study." That is one of the points of a $p$-curve disclosure table, but far from the only point. Furthermore, their selection of effects and studies to include in and exclude from their analysis was and remains unclear. Among the unsuccessful replications they included, they had doublecounting and ignored significant methodological deviations from the original experiments that were noted by the replication authors (e.g., Fayard et al., 2009; Camerer et al., 2018). They made no attempt to obtain data from successful replications they should have included (Moscatiello \& Nagel, 2014), and erroneously claimed that the link to the source produced an error. It does not. This can easily be verified by clicking on the link provided in our target article (p. 15), pasted here:

http://cupola.gettysburg.edu/cgi/viewcontent.cgi?article=1592\&context=celebration. They included some conceptual/extended replications cited in our target article but excluded other conceptual/extended replications that were also cited in our target article and also published (e.g., Denke et al., 2014; Schaefer, Rotte, Heinze, \& Denke, 2015). In

\footnotetext{
${ }^{3}$ We wrote in our target article (Lee \& Schwarz, 2021, p. 6), "A conceptual replication with an American sample showed the same pattern and also found that it was moderated by individual differences (De Los Reyes, Aldao, Kundey, Lee, \& Molina, 2012)." The original two-way interaction effect that demonstrated washing away postdecisional dissonance was moderated by individual differences, hence a three-way interaction.
} 
short, Ropovik and IJzerman's claims that they "identified all publicly available empirical evidence that Lee and Schwarz verbally presented" and that they "simply took all the studies that [Lee and Schwarz] identified as successful replications" (italics and boldface original) were erroneous.

Because of these missed steps and erroneous claims, Ropovik and IJzerman's conclusion is unwarranted. In contrast, when we followed all best practice guidelines in conducting a $p$ curve analysis, we found that the effects addressed by Ropovik et al. show evidential value. This is true regardless of whether the analysis includes only the replication studies in Ropovik et al.'s table (Figure RA1 in our response appendix) or includes those replication studies together with the original experiments being replicated (Figure RA2). The presence of evidential value is robust to exclusion of the lowest and highest $p$-values (Figure RA3). All figures are pasted at the end of this document for interested readers.

\section{Illustrative evidence is not and should not be treated as comprehensive evidence}

In addition to technical problems, we should address a more fundamental aspect of Ropovik et al.'s commentary and Ropovik and IJzerman's blog post, namely, how the illustrative evidence presented in a theory paper (our target article) should and should not be treated.

It helps to be clear about the nature of our target article and the scope of evidence that bears on it. Our target article was a theory paper that proposed the notion of grounded procedures as a proximate mechanism for (1) cleansing effects, (2) non-cleansing effects that involve physical separation, and (3) other effects that involve physical connection. From the notion of grounded procedures, we derived a set of theoretical properties. For each theoretical property, we reviewed illustrative evidence that taps into the three categories of effects. In this context, the goal of reviewing evidence was to illustrate the theory, not to provide a comprehensive coverage of all evidence available out there. The latter would be the goal of a comprehensive meta-analysis. Indeed, a separate metaanalytic review of just the first category of effects (cleansing effects) has obtained over 500 effect sizes documented in more than 200 experiments. Our view is that it would have been undesirable and infeasible to review all of these effects in our theory paper. It would have been even more undesirable and infeasible to review all available evidence for all three categories of effects. Illustrative evidence was thus our approach in the target article.

Among the spectrum of illustrative evidence presented in our target article, Ropovik et al.'s $p$-curve analysis focused on the first category of effects (cleansing effects), excluded all original experiments, included some of the replications we presented but excluded other replications we also presented that they could and should have included (as described in part 1 above), and ended up with 7 effects. Despite the narrow scope of this subset of effects in their $p$-curve analysis, their claims were much broader. The title of their commentary was "The lack of robust evidence for cleansing effects." This represents their first inferential leap, from 7 effects to all cleansing effects. The concluding line in the abstract of their commentary was "Absent more robust evidence, there is no need to develop a theoretical account of grounded procedures." This represents their second 
inferential leap, from cleansing effects to all categories of effects that illustrate grounded procedures. These leaps are unwarranted. They are problematic because, as Ropovik and IJzerman write in their blog post, "authors cannot make their set of inferences a 'moving target."' Problematic inferences, together with technical errors in their $p$-curve analysis, are the reasons for which we consider the claims in Ropovik et al.'s commentary overstated.

To be fair, it is difficult to draw broad conclusions from a small number of effects, be it the 7 effects in Ropovik et al.'s $p$-curve analysis or the 7 or 9 effects in our $p$-curve analyses (Figures RA1 and RA2). In such small sets, a few effects can have a relatively large influence on the shape of the $p$-curve, and once a $p$-curve is plotted, it is easy to forget what went into it and tempting to conclude that it simply, fully, and accurately captures reality. To guard ourselves against these, when we responded to Ropovik et al., we pointed out (in section RA.2. Scope of data considered) that it would be more informative to take a comprehensive meta-analytic approach, which has the advantages of encompassing the entire body of cleansing effects (over 500 effect sizes from over 200 experiments) and taking into account both significant and non-significant effects (as opposed to only significant effects in $p$ curve). It was and still is our stance that a quantitative presentation of the meta-analytic findings - as opposed to the qualitative summary of them in our theory paper - is the right place for the kind of quantitative discussion that Ropovik et al. were interested in.

Why did we summarize the meta-analytic finding qualitatively rather than present them quantitatively in our target article? Because it was intended as a theory paper, not a comprehensive quantitative review paper. Ropovik and IJzerman correctly note in their blog post that we "did not give [them] access to the data underlying [our] in-progress metaanalysis." (For clarity, the ongoing meta-analysis is conducted by Lee and his other collaborators, and it was appropriate that IJzerman had requested the meta-analytic dataset from Lee only.) They also correctly note that my (Lee's) reason for declining their request was to reserve the opportunity to submit to another journal a proper quantitative presentation of the meta-analysis that took several years of work to prepare. The correspondence deserves clarification. The best way to do so is to simply share my reply to IJzerman's emails.

Upon receiving $B B S^{\prime}$ s call for proposals of commentary on our target article, IJzerman emailed me to request our meta-analytic dataset so that they could analyze it thoroughly. My full reply pertaining to this request (after removing irrelevant content such as personal greetings) was as follows:

Thanks for your and Ivan's interest in the article.... Let me provide a bit of context. In the initial submission, we didn't have the part where we now provide a metaanalytic perspective on this area of work (section 1.2). In the review process, we were requested to provide some reason for our belief in the empirical foundation of this area of work. Because I had been (and still am) writing up a meta-analytic review with my other coauthors, I checked with the target journal for the meta-an (Psych Bull, no surprise) and learned that the best way to proceed was to describe the insights qualitatively and avoid presenting quantitative results in the present article, hence our decidedly qualitative approach to it ("Full results are beyond the 
scope of this article, but a few observations are relevant and summarized qualitatively here." on pp. 10-11 [of the manuscript]). The rest of the article proceeded to theoretical treatment of the subject matter, which was the primary focus of the present piece.

Because the meta-analytic review is still being written up, and any quantitative presentation of its results would prevent my other coauthors and me from submitting to Psych Bull, I hope it's understandable that it's not yet shareable at the moment, but it will be shareable later. Whichever journal ends up taking our metaanalysis, I hope and I think, will be the place and time for the quantitative dialogue. If we're lucky enough to get it into Psych Bull, I know for sure that commentary will be welcome. In fact, you've already published commentary in Psych Bull before, so you know the process even better than I do. In addition to that, it does sound like your workflow has much to offer. When it becomes shareable, I'd very much like to learn from it and apply it to our ongoing work too.

So, again, my apologies for the timing, but I hope it's understandable.

IJzerman sent me a follow-up request, to which I replied as follows.

Thanks for taking the time to offer your additional thoughts and suggestion, which I do appreciate. Sorry I should have been clearer in the last email regarding the Psych Bull requirement: quantitative presentation of the meta-analytic results has to first appear there, or else we would be ineligible, which was why in the BBS piece we focused on a qualitative description of the meta-analytic perspective on this area of work, saving the actual quantitative results for another submission. The implication is that by sharing the data we extracted and coded over the past few years for this meta-analysis and having the results in print anywhere (by you or us or others), we wouldn't be able to submit it. In other words, it's not that I'm worried about finding out weak results that would undermine our chances at Psych Bull, but that it would altogether disqualify us from submitting there.

I recognize the tension between our perspectives. Taking a step back, it seems to boil down to where the quantitative details are to be grappled with. To the extent that we only provided a qualitative description and did so briefly in 2.5 out of 47 [manuscript] pages of a fundamentally theoretical piece that covers a range of phenomena from cleansing to separation to touch to approach/avoidance and more, intently saving the quantitative results for the other manuscript that awaits completion, the journal for the latter seems to be the appropriate place, where I really think the most productive empirical discussion can happen. I look forward to that."

For these reasons, I did not share the meta-analytic dataset. It remains our view that a proper quantitative presentation of the comprehensive evidence in the form of a metaanalysis - as opposed to the qualitative summary of illustrative evidence in our theory paper-is the right place for more fruitful quantitative discussion. 


\section{Conclusion}

We appreciate Ropovik and IJzerman's interest in our target article and authors' response. Their blog post highlights two aspects in which we disagree with them. First, when implementing a $p$-curve analysis, we consider it crucial to properly follow all best practices, not just selectively follow some of them. Second, it is our stance that illustrative evidence in a theory paper should not be treated as if it were comprehensive coverage of all available evidence.

\section{References}

Albarracín, D., Cuijpers, P., Eastwick, P. W., Johnson, B. T., Roisman, G. I., Sinatra, G. M., \& Verhaeghen, P. (2018). Editorial. Psychological Bulletin, 144(3), 223-226. https://doi.org/10.1037/bul0000147

Camerer, C. F., Dreber, A., Holzmeister, F., Ho, T.-H., Huber, J., Johannesson, M., Kirchler, M., Nave, G., Nosek, B. A., Pfeiffer, T., Altmejd, A., Buttrick, N., Chan, T., Chen, Y., Forsell, E., Gampa, A., Heikensten, E., Hummer, L., Imai, T., ... Wu, H. (2018). Evaluating the replicability of social science experiments in Nature and Science between 2010 and 2015. Nature Human Behaviour, 2(9), 637-644. https://doi.org/10.1038/s41562018-0399-Z

De Los Reyes, A., Aldao, A., Kundey, S. M. A., Lee, B. G., \& Molina, S. (2012). Compromised decision making and the effects of manipulating physical states on human judgments. Journal of Clinical Psychology, 68(1), 1-7. https://doi.org/10.1002/jclp.20851

Denke, C., Rotte, M., Heinze, H.-J., \& Schaefer, M. (2014). Lying and the Subsequent Desire for Toothpaste: Activity in the Somatosensory Cortex Predicts Embodiment of the Moral-Purity Metaphor. Cerebral Cortex, 26(2), 477-484. https://doi.org/10.1093/cercor/bhu170

Fayard, J. V., Bassi, A. K., Bernstein, D. M., \& Roberts, B. W. (2009). Is cleanliness next to godliness? Dispelling old wives' tales: Failure to replicate Zhong and Liljenquist (2006). Journal of Articles in Support of the Null Hypothesis, 6(2), 21-29.

IJzerman, H., Hadi, R., Coles, N., Sarda, E., Klein, R. A., \& Ropovik, I. (2020). Social thermoregulation: A meta-analysis. Unpublished manuscript.

Lee, S. W. S., \& Schwarz, N. (2021a). Grounded procedures: A proximate mechanism for the psychology of cleansing and other physical actions. Behavioral and Brain Sciences, 44, e1. https://doi.org/10.1017/S0140525X20000308

Lee, S. W. S., \& Schwarz, N. (2021b). Grounded procedures in mind and society. Behavioral and Brain Sciences, 44, e29. https://doi.org/10.1017/S0140525X20000643

Moscatiello, T., \& Nagel, E. (2014, May 2). The effects of cleansing on risk taking [Poster]. Celebration, Gettysburg, PA.

http://cupola.gettysburg.edu/cgi/viewcontent.cgi?article=1592\&context=celebration

Ropovik, I., Sparacio, A., \& IJzerman, H. (2021). The lack of robust evidence for cleansing effects. Behavioral and Brain Sciences, 44, e18. https://doi.org/10.1017/S0140525X20000448

Schaefer, M., Denke, C., Heinze, H.-J., \& Rotte, M. (2014). Rough primes and rough conversations: Evidence for a modality-specific basis to mental metaphors. Social 
Cognitive and Affective Neuroscience, 9(11), 1653-1659.

https://doi.org/10.1093/scan/nst163

Simonsohn, U., Nelson, L. D., \& Simmons, J. P. (2014). P-curve: A key to the file-drawer. Journal of Experimental Psychology: General, 143(2), 534-547. https://doi.org/10.1037/a0033242

Simonsohn, U., Simmons, J. P., \& Nelson, L. D. (2015). Better P-curves: Making P-curve analysis more robust to errors, fraud, and ambitious P-hacking, a Reply to Ulrich and Miller (2015). Journal of Experimental Psychology: General, 144(6), 1146-1152. https://doi.org/10.1037/xge0000104

Sparacio, A., Ropovik, I., Jiga-Boy, G. M., Forscher, P. S., Paris, B., \& IJzerman, H. (2021). Stress regulation via self-administered mindfulness and biofeedback interventions in adults: A pre-registered meta-analysis. Unpublished manuscript. 


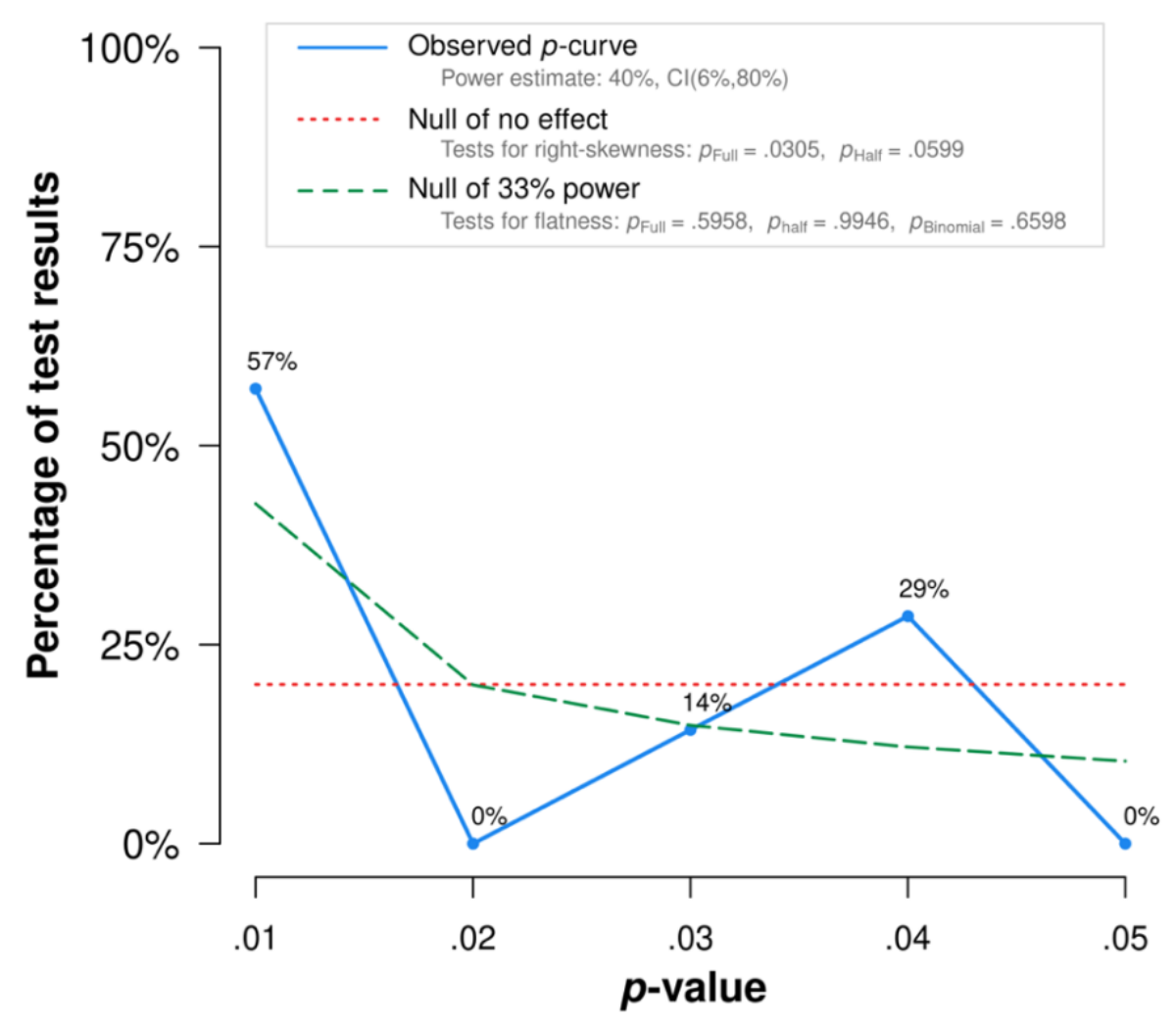

Note: The observed $p$-curve includes 7 statistically significant $(p<.05)$ results, of which 5 are $p<.025$. There were no non-significant results entered.

\begin{tabular}{|c|c|c|c|}
\hline \multirow[b]{3}{*}{$\begin{array}{l}\text { 1) Studies contain evidential value. } \\
\text { (Right skew) }\end{array}$} & \multirow[t]{2}{*}{$\begin{array}{l}\text { Binomial Test } \\
\text { (Share of results } p<.025 \text { ) }\end{array}$} & \multicolumn{2}{|c|}{$\begin{array}{l}\text { Continuous Test } \\
\text { (Aggregate with Stouffer Method) }\end{array}$} \\
\hline & & $\begin{array}{l}\text { Full p-curve } \\
\left(p^{\prime} s<.05\right)\end{array}$ & $\begin{array}{l}\text { Half p-curve } \\
\left(p^{\prime} s<.025\right)\end{array}$ \\
\hline & $p=.2266$ & $Z=-1.87, p=.0305$ & $Z=-1.56, p=.0599$ \\
\hline \multirow[t]{2}{*}{$\begin{array}{l}\text { 2) Studies' evidential value, if any, is } \\
\text { inadequate. } \\
\text { (Flatter than } 33 \% \text { power) }\end{array}$} & $p=.6598$ & $Z=0.24, p=.5958$ & $Z=2.55, p=.9946$ \\
\hline & \multicolumn{3}{|c|}{ Statistical Power } \\
\hline $\begin{array}{l}\text { Power of tests included in } p \text {-curve } \\
\quad \text { (correcting for selective reporting) }\end{array}$ & \multicolumn{3}{|c|}{$\begin{array}{c}\text { Estimate: } 40 \% \\
\text { 90\% Confidence interval: }(6 \%, 80 \%)\end{array}$} \\
\hline
\end{tabular}

\section{Interpretation:}

$P$-Curve analysis combines the half and full $p$-curve to make inferences about evidential value. In particular, if the half $\mathrm{p}$-curve test is right-skewed with $p<.05$ or both the half and full test are right-skewed with $p<.1$, then $p$-curve analysis indicates the presence of evidential value. This combination test, introduced in Simonsohn, Simmons and Nelson (2015 .pdf) 'Better P-Curves' paper, is much more robust to ambitious $p$-hacking than the simple full $p$-curve test is.

Here we see that both tests are $p<.1$, indicating evidential value.

Figure RA1. P-curve of statistically significant results from replications in Ropovik et al.'s table. 


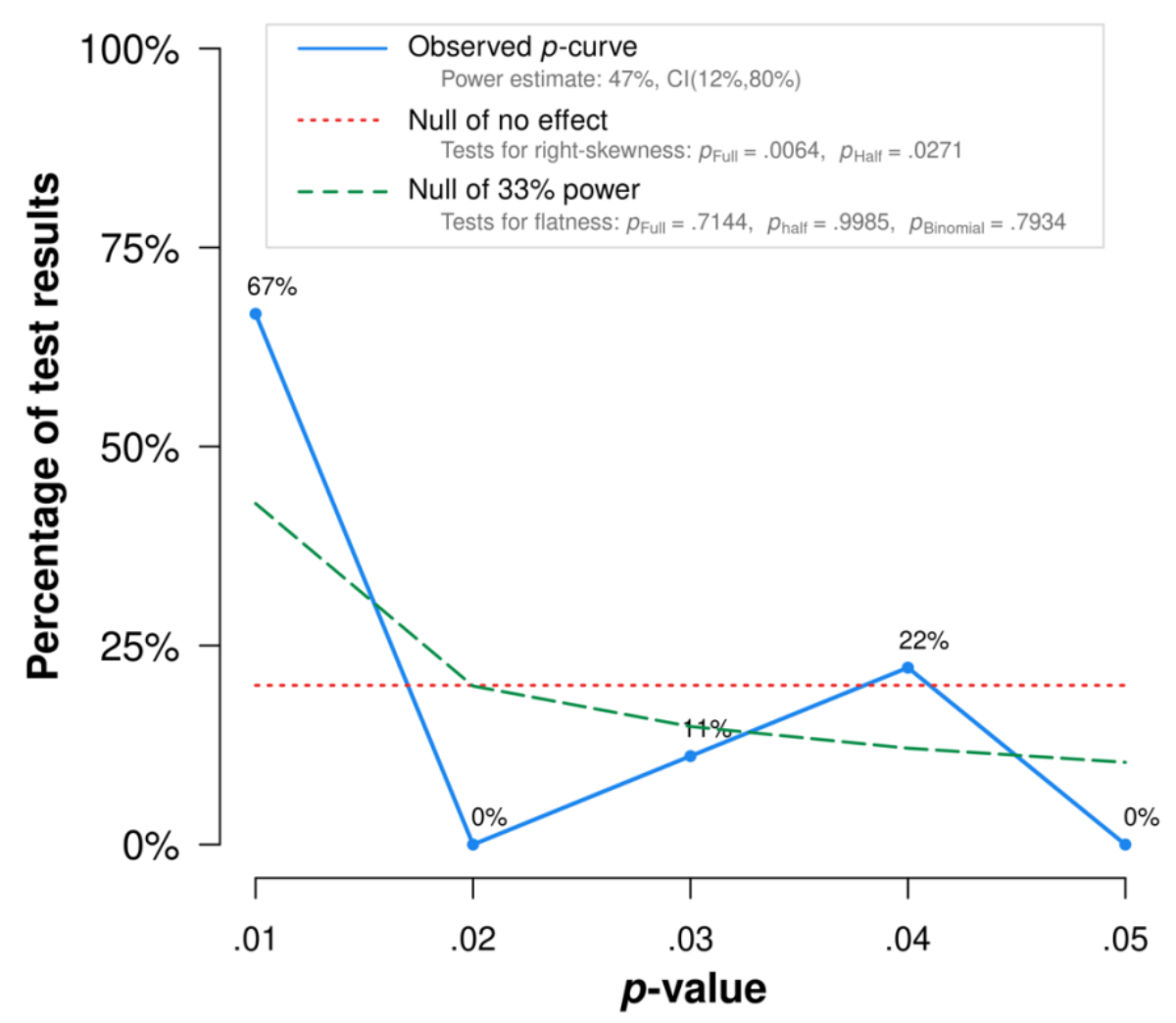

Note: The observed $p$-curve includes 9 statistically significant $(p<.05)$ results, of which 7 are $p<.025$. There were no non-significant results entered.

\begin{tabular}{|c|c|c|c|}
\hline \multirow[b]{3}{*}{$\begin{array}{l}\text { 1) Studies contain evidential value. } \\
\text { (Right skew) }\end{array}$} & \multirow{3}{*}{$\begin{array}{c}\text { Binomial Test } \\
\text { (Share of results } p<.025 \text { ) } \\
p=.0898\end{array}$} & \multicolumn{2}{|c|}{$\begin{array}{l}\text { Continuous Test } \\
\text { (Aggregate with Stouffer Method) }\end{array}$} \\
\hline & & $\begin{array}{l}\text { Full p-curve } \\
\text { (p's<.05) }\end{array}$ & $\begin{array}{l}\text { Half p-curve } \\
\text { (p's<.025) }\end{array}$ \\
\hline & & $Z=-2.49, p=.0064$ & $Z=-1.93, p=.0271$ \\
\hline \multirow[t]{2}{*}{$\begin{array}{l}\text { 2) Studies' evidential value, if any, is } \\
\text { inadequate. } \\
\text { (Flatter than } 33 \% \text { power) }\end{array}$} & $p=.7934$ & $Z=0.57, p=.7144$ & $Z=2.97, p=.9985$ \\
\hline & \multicolumn{3}{|c|}{ Statistical Power } \\
\hline $\begin{array}{l}\text { Power of tests included in p-curve } \\
\text { (correcting for selective reporting) }\end{array}$ & \multicolumn{3}{|c|}{ Estimate: $47 \%$} \\
\hline
\end{tabular}

\section{Interpretation:}

$P$-Curve analysis combines the half and full $p$-curve to make inferences about evidential value. In particular, if the half $p$-curve test is right-skewed with $p<.05$ or both the half and full test are right-skewed with $p<.1$, then $p$-curve analysis indicates the presence of evidential value. This combination test, introduced in Simonsohn, Simmons and Nelson (2015 .pdf) 'Better P-Curves' paper, is much more robust to ambitious $p$-hacking than the simple full $p$-curve test is.

Here both conditions are met, indicating evidential value.

Figure RA2. P-curve of statistically significant results from replications in Ropovik et al.'s table and the original experiments being replicated. 

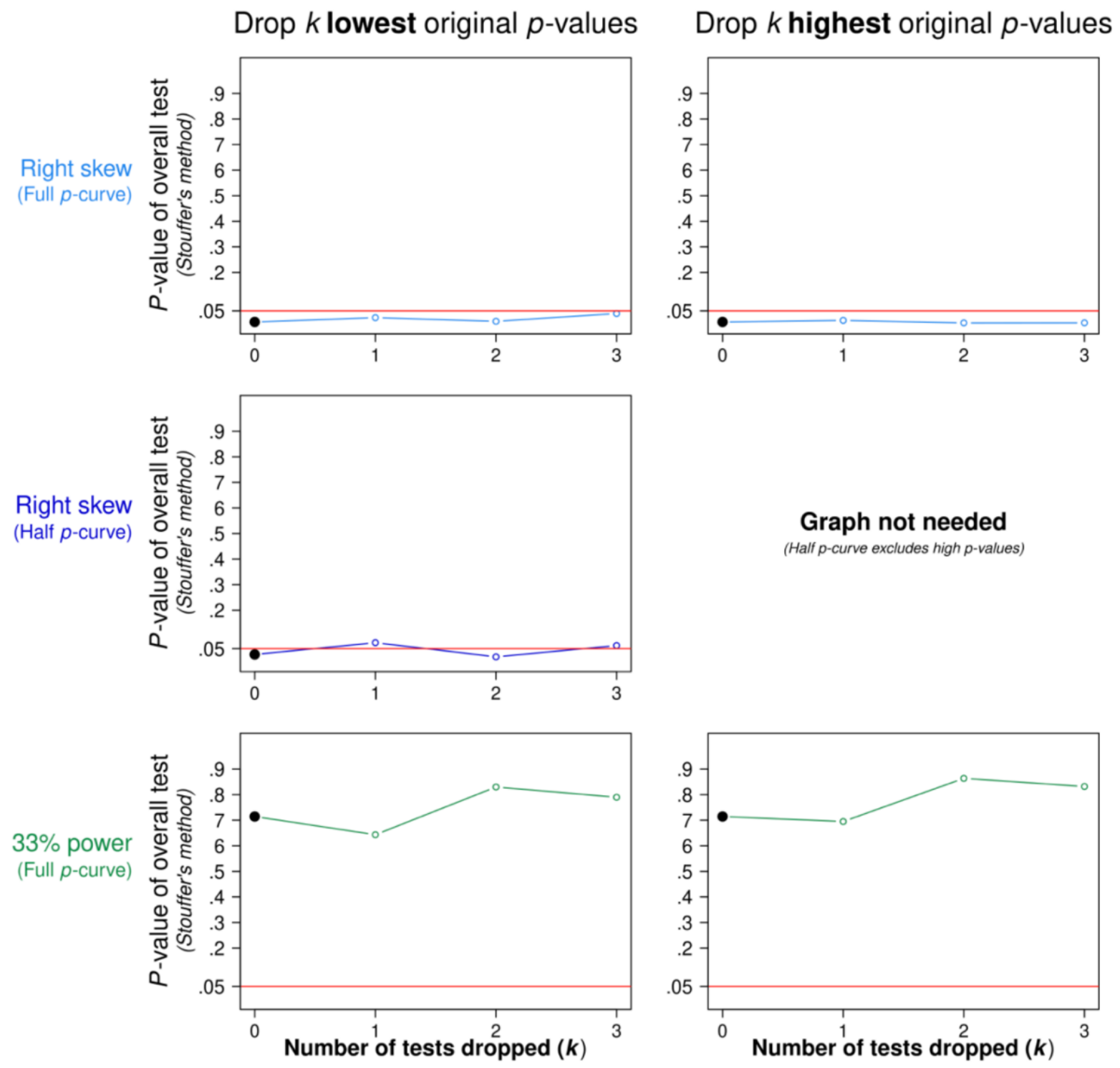

- Including all $p$-values $\circ$ Dropping $p$-values

Figure RA3. Robustness of evidential value to exclusion of lowest and highest $p$-values. As noted in the $p$-curve app (http://www.p-curve.com/app4/pcurve4.php), "We should place more confidence in sets of studies whose overall evidential value survives the exclusion of the most extreme few results." 